\title{
Performance Comparison of Two Infrared Sensor Types for Indoor Multi-Node Communication
}

\author{
Abdulmuttalib T. Rashid \\ Electrical Engineering Dept. \\ University of Basrah \\ Basrah, Iraq
}

\author{
Ola A. Hasan \\ Electrical Engineering Dept. \\ University of Basrah \\ Basrah, Iraq
}

\author{
Ramzy S. Ali \\ Electrical Engineering Dept. \\ University of Basrah \\ Basrah, Iraq
}

\begin{abstract}
This paper compares the performance of two types of IR sensors that are used for communication among nodes to get the identity of each other. Practical circuits for these sensor types are built and tested in an environment which has nodes with cylindrical shape. Each node equipped by several pair of sensors: one sensor in each pair works as a transmitter and the other works as a receiver. The communication between sensors is repeated for different angles of rotation to produce a complete performance to each sensor. Based on the results of these comparisons, the TSOP4P38 IR receiver with remote control were chosen for communication in the practical implementation.
\end{abstract}

\section{General Terms}

Communication, Identification.

\section{Keywords}

IR transmitter; IR receiver; TSOP4P38 sensor,

\section{INTRODUCTION}

An infrared sensor is an electronic device that can be used to sense its surrounding environment by either emitting or detecting infrared radiation. These characteristics give the capability to use the Infrared sensors in different fields like communication, distance or angle measurements, tracking, obstacle detection and localization [1 - 9].

In wireless sensor networks (WSN), these sensors maybe used for communication, distance or angle measurements [10-11]. The inexpensiveness and the accuracy of these sensors make them very suitable select for being used in distance measuring [12]. A mobile sensor network is collected of a distributed group of nodes, each of which has sensing, communication and localization capabilities [13]. On the other hand, not only the distance calculation is needed for localization; Localization algorithms need to know the identity of sender and receiver and some of them are depended on the connectivity among nodes to estimate the nodes' locations. Again, we are looking for inexpensive sensors to achieve communication among nodes for an indoor system which are the infrared sensors [14].

Wireless infrared communications mentions to the use of freeSpace broadcast of light waves in the close to the infrared band as a transmission medium for communication [15-16]. Infrared sensors offer several advantages over radio devices in a short-range medium and indoor communication. The Infrared transmitter and receiver capable of high speed operation and they are available at low cost. They are also offer an unlimited bandwidth and close together in wavelength, and they exhibit similar behavior [1-2].

In this paper the communication is based on design a system with several group of sensors pair: the first one of sensor pair is used as a transmitter and the other is used as a receiver. Two types of sensor pairs are used: IR sensor pair and the TSOP4P38 IR receiver with remote control. The rest of the paper is organized as follows: Section 2 explains the system description, the experimental results shown in section 3 , and finally section 4 discuss the results.

\section{SYSTEM DESCRIPTION}

This section includes description, the electronic circuits, and the schematic diagrams of two types of sensors that can be used for nodes communication. These sensors are the IR transmitter and receiver pair and the TSOP4P38 IR receiver with remote control. One sensor of each sensor pair is fixed on one node to work as a receiver and the other is fixed on other node to work as a transmitter.

\subsection{IR transmitter and receiver}

IR pair consists of IR LED and photo diode. The IR LED radiates an infrared light and the resistance of the photo diode will change depending on the amount of IR radiation that falling on it. There are two ways to place the IR pair which are: direct and indirect incidences. During the direct incidence as in Fig. 1, the IR LED will be placed in front of the photo diode. So, the infrared signal will be directly sensed by the photo diode and placing any object between them will cause to prevent the access of IR signal to the receiver. On the other hand, putting the photo diode and IR LED in parallel to each other facing the same direction will result into an indirect incidence. In this case, if the IR signal is reflected by some object then the reflected light will be absorbed by the photo diode. Fig. 2 shows the case of indirect incidence.

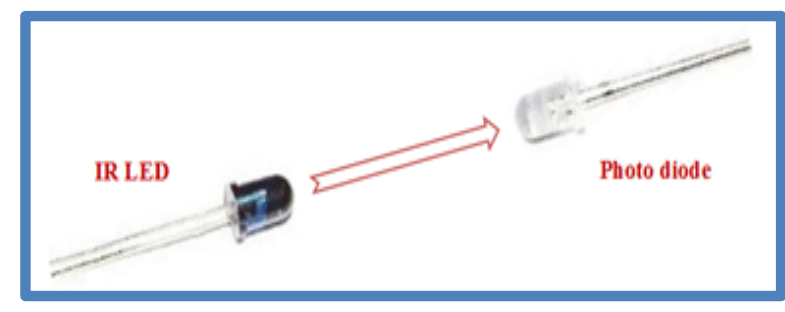

Fig. 1. The direct incidence between the IR pair

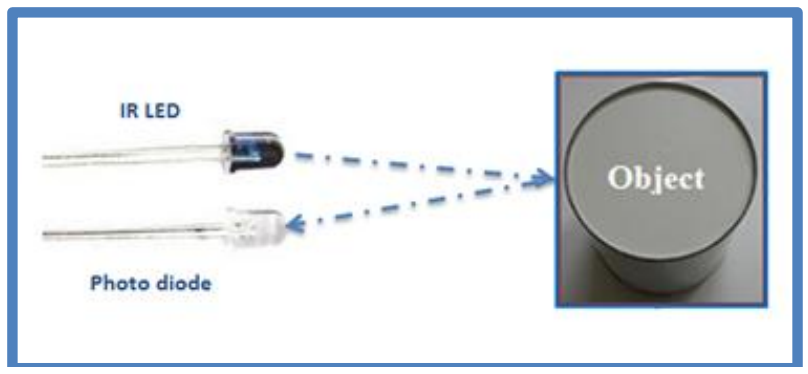

Fig. 2. The indirect incidence between the IR pair 
Fig. 3 shows that we had used the $5 \mathrm{~mm}$ IR pair in which has wave length of $940 \mathrm{~nm}$. The one of blue lens color represents the IR LED while the one with water clear lens color acts as a photo diode [17].

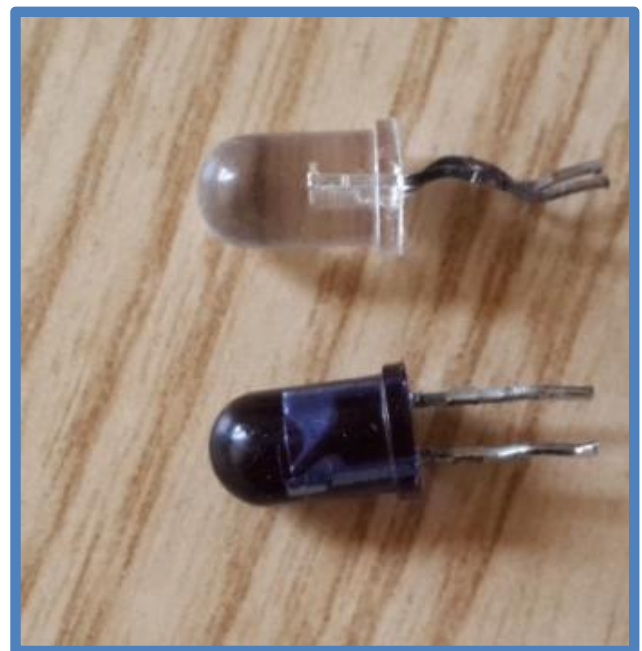

Fig. 3. The 5mm IR transmitter and receiver

\subsection{The TSOP4P38 IR with remote control}

Also, we shall use another type of IR receiver which is TSOP4P38 as in Fig. 4. This receiver is acting as a preamplifier and photo detector in one package; it has an Internal filter for burst frequency and needs input voltage from $2.7 \mathrm{~V}$ to $5.5 \mathrm{~V}$ to operate probably [18].

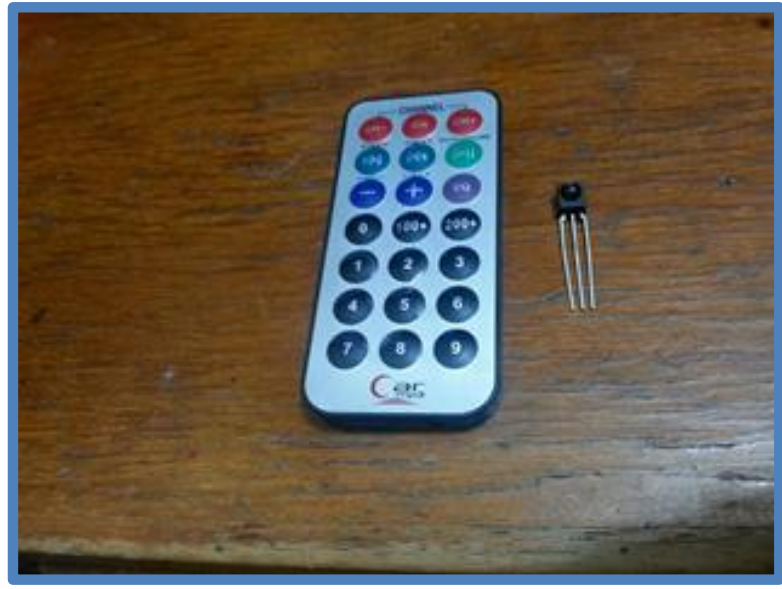

Fig. 4. The TSOP4P38 IR receiver with a remote control

\section{EXPERIMENTAL RESULTS}

The following experiments include a data collection system which is used for investigate the communication between any two nodes. The experiments are implemented into two systems: one of them uses $5 \mathrm{~mm}$ IR transmitter and receiver system while the other one uses TSOP4P38 receiver with a remote control.

In Fig. 5 both of the $5 \mathrm{~mm}$ IR transmitter and receiver are connected through $100 \Omega$ resistor to a separate arduino kit. The schematic diagram is shown in Fig. 6.

Fig. 7 shows how the TSOP4P38 receiver is connected to the arduino kit, this type of IR receives the remote control signal in all directions ( $360^{\circ}$ viewing angle). So, we put a shield on the sensor to reduce its viewing angle. The schematic diagram
TSOP4P38
is
illustrated
in Fig.

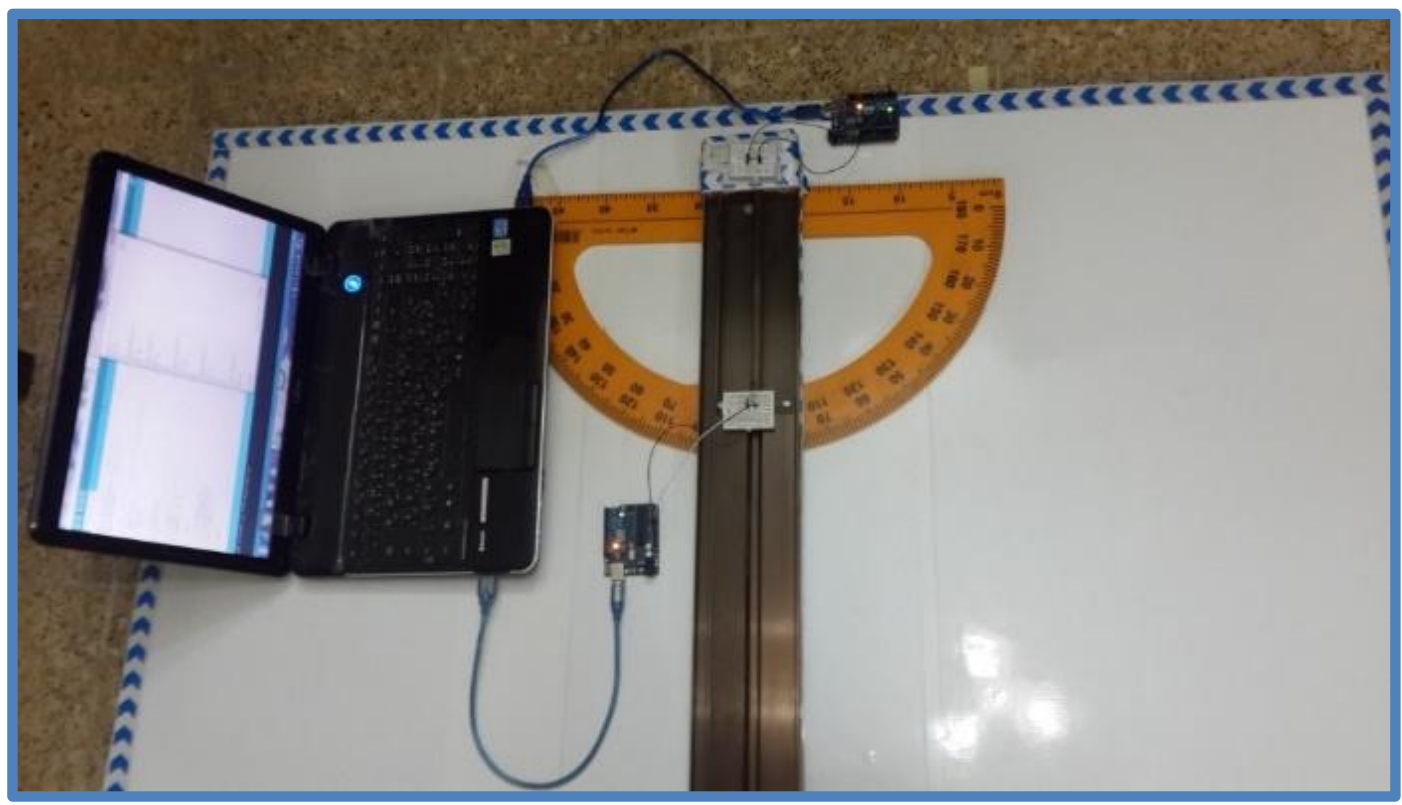

Fig. 5. Connecting $5 \mathrm{~mm}$ IR transmitter and receiver to Arduino 


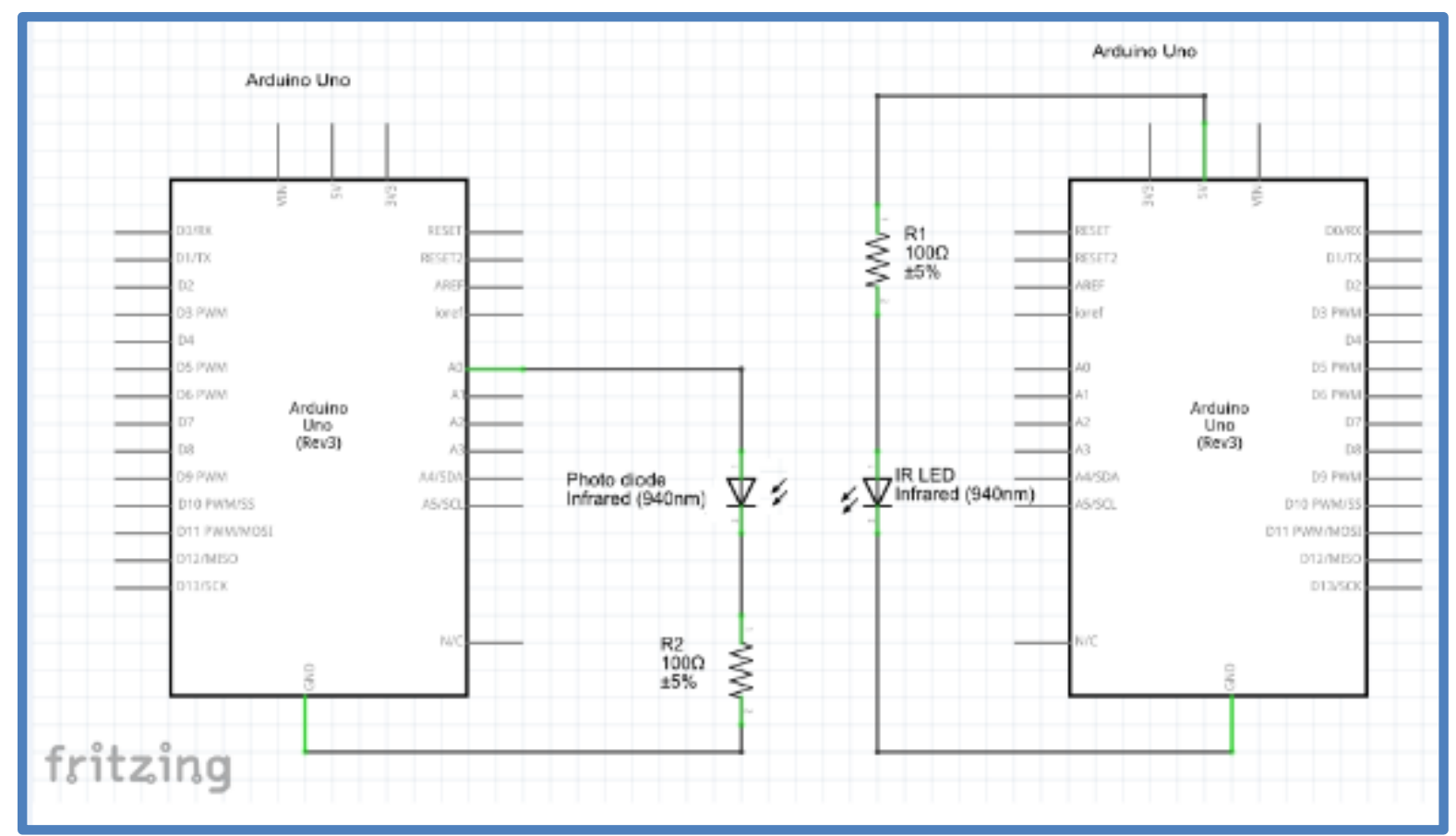

Fig. 6. Schematic diagram of $5 \mathrm{~mm}$ IR transmitter and receiver

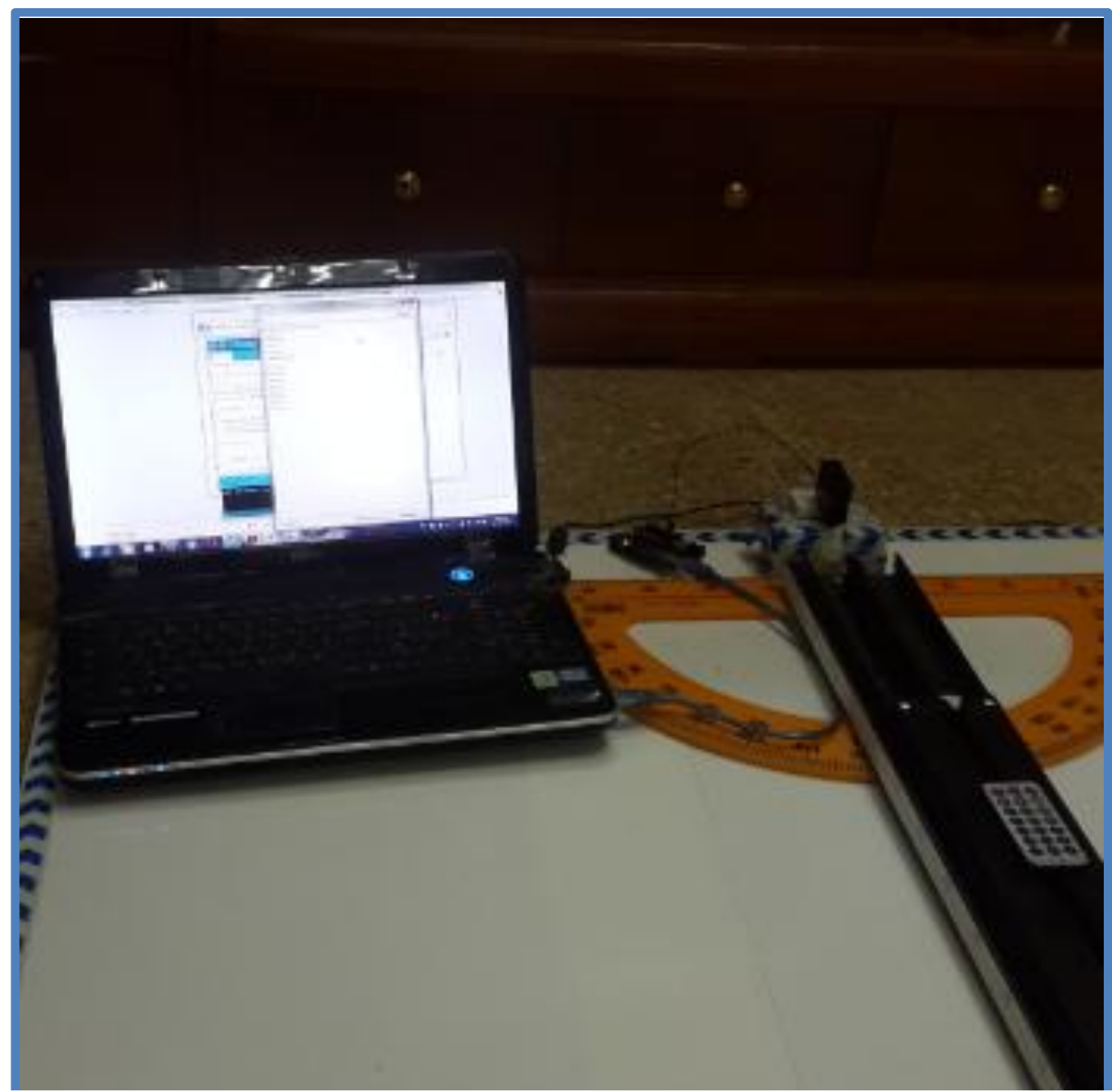

Fig. 7. Attaching TSOP4P38 IR sensor with a shield to arduino 


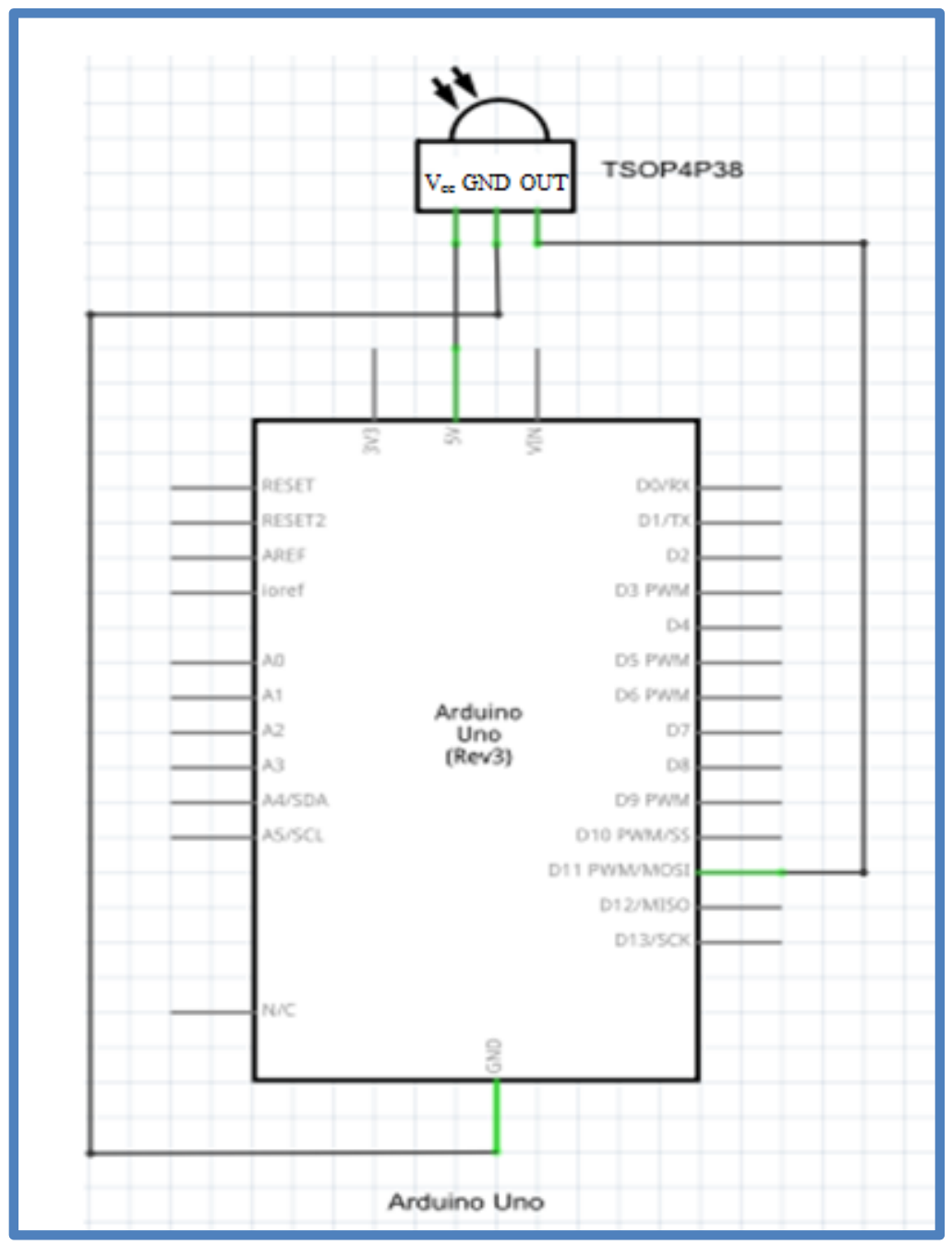

Fig. 8. TSOP4P38 IR receiver schematic diagram

The experiment begins by placing the IR receiver on a fixed part on board and placing the IR sender on the arm sliding part in a line of sight with the receiver, then starts to rotate the arm by a step of $10^{\circ}$ each time and measures the maximum distance at which the sender and receiver can communicate. The curves of both systems in Fig. 9 show that the maximum distance at an angle of $0^{\circ}$ will be about six and a half meters when using the TSOP4P38 sensor and it will be about one meter for the $5 \mathrm{~mm}$ IR sensor. Again the curves show that the largest angle among the sender and receiver and still able to communicate with each other is $60^{\circ}$ for TSOP4P38 with a shield and $30^{\circ}$ for $5 \mathrm{~mm}$ IR pair. Fig. 10 shows how many sensors are needed to cover $360^{\circ}$ circular surface. Where the red sketch represents the $5 \mathrm{~mm}$ IR receiver and the blue one represents the TSOP4P38 sensor.

\section{CONCLUSION}

This paper is focused on choosing some sensors to achieve communication among nodes and indicate the identities of them. For the communication among nodes, we noticed that the TSOP4P38 receiver with remote control is better than $5 \mathrm{~mm}$ IR transmitter and receiver in the term of distance where the maximum distance that the remote control and TSOP4P38 receiver can communicate with other is about $6 \mathrm{~m}$ while for the $5 \mathrm{~mm}$ IR pair is about $150 \mathrm{~cm}$. As well as the remote control has many codes that can take advantage of them as identities for nodes. 


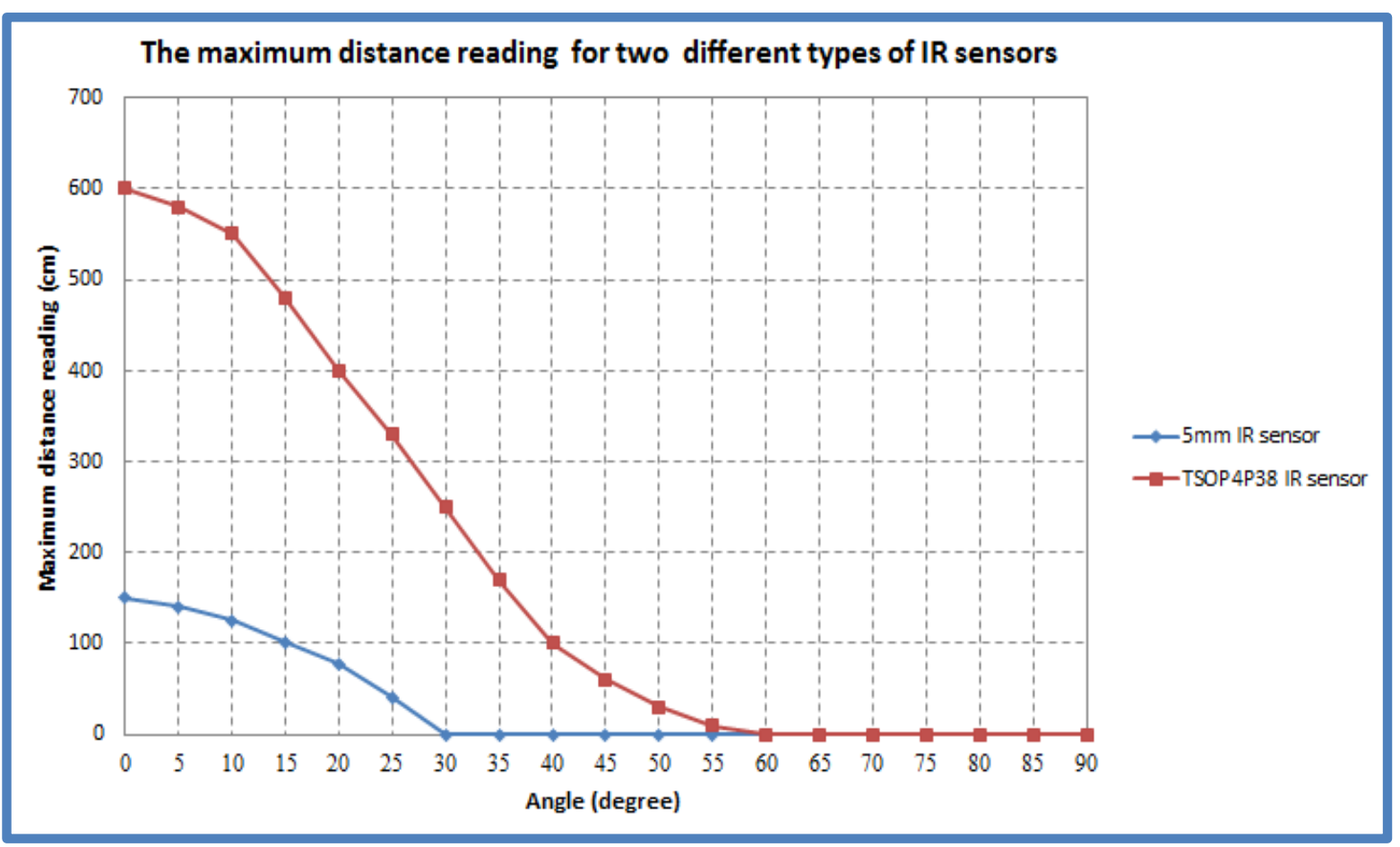

Fig. 9. The maximum distance reading at different angles for both of $5 \mathrm{~mm}$ IR and TSOP4P38 IR sensors

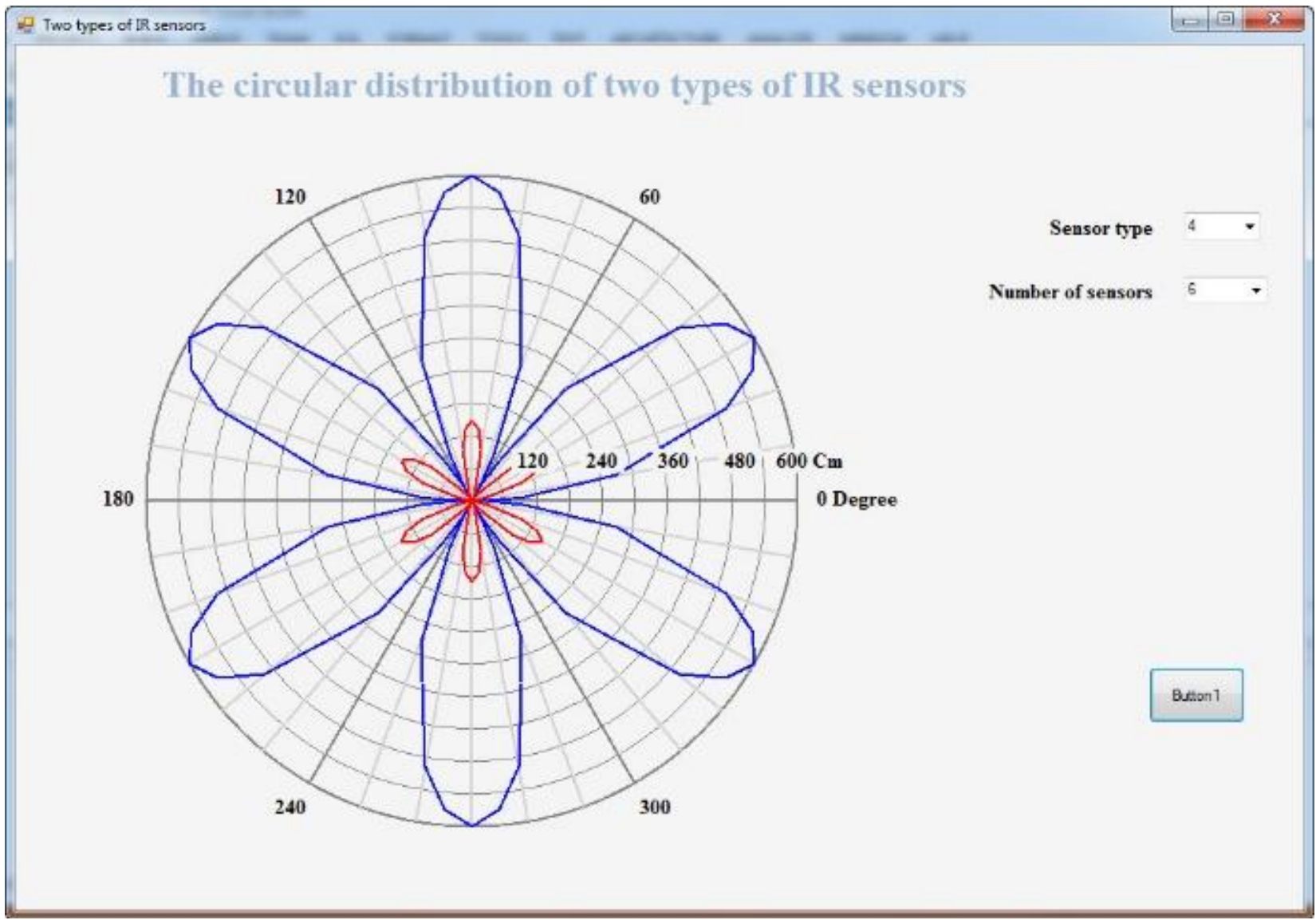

Fig. 5.30 The circular distribution for two types of IR sensors 


\section{REFERENCES}

[1] F. R. Gfeller and U. H. Bapst, "Wireless in-house data communication via diffuse infrared radiation," Proc. IEEE, vol. 67, pp. 1474-1486, Nov. 1979.

[2] Y. Nakata, J. Kashio, T. Kojima, and T. Noguchi, "Inhousewireless communication system using infrared radiation," Proc.7th Int. Conf. on Computer Commun., Sydney, Australia, Oct.30-Nov. 2, 1984, pp. 333-337.

[3] A. T. Rashid, M. Frasca, A, A, Ali, A. Rizzo and L. Foruna," Multi-robot localization and orientation estimation using robotic cluster matching algorithm. Robotics and Autonomous Systems, Vol. 63, p.p. 108121, 2015.

[4] A. T. Rashid, W. H. Zayer and M. T. Rashid," Design and Implementation of Locations Matching Algorithm for Multi-Object Recognition and Localization", Iraqi Journal of Electrical and Electronic Engineering, Vol. 14, No. 1, p.p. 10-21, 2018.

[5] O. A. Hasan, A. T. Rashid, R. S. Ali and J. Kosha," A Practical Performance Analysis of Low-Cost Sensors for Indoor Localization of Multi-Node Systems “, Internet Technologies and Applications (ITA), Wrexham UK, September 2017.

[6] O. A. Hasan, A. T. Rashid and R. S. Ali," Centralized approach for multi-node localization and identification ", Iraq J. Electrical and Electronic Engineering, Vol.12 No. 2, pp. 178-187, 2016.

[7] O. A. Hasan, A. T. Rashid and R. S. Ali," A Hybrid approach for multi-node localization and Identification ", Basrah Journal for Engineering Sciences, vol. 16, no. 2, pp. 11- 20, 2016.

[8] A. T. Rashid and A. A. Ali, "Performance Analysis of Low-Cost Infrared Sensors for Multi-Robot Localization and Communication", International Journal of Computer Applications, vol. 182, No. 26, pp. 23-29, 2018.

[9] I. S. Alfurati and A. T. Rashid, "Performance Comparison of Three Types of Sensor Matrices for Indoor Multi-Robot Localization", International Journal of Computer Applications, vol. 181, No. 26, pp. 22-29, 2018 .

[10] Y. T. Win, H. T. Htun, N. Afzulpurkar and C. Punyasai , Ultrasonic System Approach to Obstacle Detection and Edge Detection", Sensors \& Transducers Journal, Vol. 127, Issue 4, pp. 56-68, 2011.

[11] B. Mustapha, A. Zayegh1and R.K. Begg,' Microcontroller Based Wireless Obstacle Detection System for the Elderly", 2014 4th International Conference on Artificial Intelligence with Applications in Engineering and Technology, 2014.

[12] M. R. Yaacob, N. S. N. Anwar, and A. M. Kassim, "Effect of glittering and reflective objects of different colors to the output voltage-distance characteristics of sharp GP2D120 IR,” ACEEE Int. J. on Electrical and Power Engineering, vol. 03, no. 02, 2012.

[13] A. Howard, M. J Mataric, and G. S Sukhatme," Mobile Sensor Network Deployment using Potential Fields: A Distributed, Scalable Solution to the Area Coverage Problem", H. Asama et al. (eds.), Distributed Autonomous Robotic Systems 5 (C) Springer-Verlag Tokyo 2002.

[14] [M, Sharma, A. Grover and P. Bande,'Low Cost Sensors for General Applications", International Journal of Recent Trends in Engineering, Vol, 1, No. 5, pp. 150$152,2009$.

[15] F. R. Gfeller and U. H. Bapst, IWireless in-house data Communication via diÆuse infrared radiation," ProceedCARR UTHERS: WIRELESS INFRARED COMMUNICA TIONS 7 ings of the IEEE, vol. 67, pp. $1474\{1486$, Nov. 1979.

[16] J. M. Kahn and J. R. Barry, IWireless infrared communications," Proceedings of the IEEE, vol. 85, pp. $265\{98$, Feb. 1997.

[17] 5 mm infrared LED, T1-1 3/4 IR333, Technical Data Sheet, 2005 .

[18] IR receiver modules for mid-range proximity sensors (TSOP4P) datasheet, Vishay Semiconductors, 2010. 